Tatjana Samardžija

Univerzitet u Beogradu

Filološki fakultet

tatjana.g.samardzija@gmail.com

https://doi.org/10.18485/ai_savremeni_roman.2020.ch6

821.133.1.09-31 Houellebecq M.

Оригинални научни рад

\title{
LA TRIPLE CASTRATION DE L'HOMME DANS SÉROTONINE DE MICHEL HOUELLEBECQ
}

Larticle analyse la triple castration deros et poiesis (force sexuelle et créatrice) de l'homme occidental que présente Sérotonine. De nombreuses citations, lexèmes et sèmes récurrents montrent que cette castration se manifeste aux niveaux pharmaceutique (antidépresseurs qui ne soignent pas la dépression, mais causent l'impuissance sexuelle et intensifient l'isolement social de l'individu souffrant), idéologique (transformation idéologique des rapports homme-femme qui pèse lourd sur les relations intimes et familiales) et socio-politique (mesures politiques et juridiques qui sapent l'autorité de l'homme dans la famille, découragent lesprit entrepreneur dans léconomie et afaiblissent le lien entre l'homme et le sol natal).

Mots-clés: castration, christianisme, hétérosexuel, Houellebecq, Huxley, impuissance, libéralisme, féminisme, Occident, sérotonine.

\section{Introduction}

A l'instar des romans précédents de Michel Houellebecq $(\mathrm{MH})$, qui offrent un panorama multidimensionnel de l'entropie éthique et psychologique atomisant la structure de la personnalité individuelle et de la société française et 
occidentale, Sérotonine (2018) est l'autorécit d’un Français d'âge moyen, " gueux d'amour ${ }^{18}$ solitaire, incapable d'aimer mais rêvant de l'amour inconditionnel de la Femme dans une société de plus en plus hostile et complexe.

Malgré la présence de plusieurs constantes houellebecquiennes dans le roman, une première grande différence qu'introduit Sérotonine est la disparition de la cuirasse cynique qui protégeait l'anti-héros du monde et de la vie ; cette fois, Florent-Claude Labrouste est ouvertement sensible, quitte à être pathétique. Deuxièmement, Sérotonine remplace les séquences pornographiques des romans précédents par la disparition progressive de la libido chez l'anti-héros. En même temps, paradoxalement, Florent croit plus que jamais à l'amour : Le monde extérieur était dur, impitoyable aux faibles, il ne tenait presque jamais ses promesses, et l'amour restait la seule chose en laquelle on puisse encore, peut-être, avoir foi. (180)

Toutefois, l'auteur garde la conviction que la sexualité physique - mais, cette fois, avec la femme aimée, sans promiscuité - est le déclencheur et l'essence de l'amour et du bonheur de l'homme. La troisième nouveauté - notamment, par rapport aux Particules élémentaires (1998, PE), La possibilité d'une île (2005, PI) et La carte et le territoire (2010, CT) - le narrateur, notre contemporain, nest pas dédoublé d'un autre, situé dans un avenir dystopique plus ou moins ultérieur.

Le titre du roman est une sorte d'antiphrase : Florent souffre du manque d'hormone sérotonine (elle contribue à

18 La chanson du gueux d'amour » de J. Jacquin (paroles) et J. Soulacroix (musique) de 1920 a inspiré notre traduction de la collocation serbe тьубавна сиротиюа (litt. " le(s) misérable(s) d’amour ") que nous devons au poète, chanteur et compositeur serbe Bora Đorđević. (v. Samardžija 2018). Retrouver "La chanson du gueux d'amour" sur https://gallica.bnf.fr/ark:/12148/bpt6k382129g.image 10.10.19. 
assurer une position sociale et sexuelle dominante), et le médicament censé y remédier provoque son impuissance sexuelle et sociale totale. Même Aymeric, l'ami de Florent, qui, au début, affiche une sérotonine triomphante, reste seul, écrasé par les conséquences socio-économiques des mesures globalisantes de l'Union européenne. Leurs destins complémentaires accusent la société et son idéologie dominante de châtrer l'homme occidental de manières socio-politique, idéologique ${ }^{19}$ et même pharmaceutique. C'est cette triple castration de l'homme occidental que nous nous proposons d'étudier dans la présente analysé. Castration? Mot trop fort ? Non, puisque Sérotonine dénonce ouvertement différents menaces contre la (pro)création (eros et poiesis) de l'homme français et occidental.

\section{La triple castration dans Sérotonine}

Les destins entrelacés de Florent et Aymeric, lesquels se terminent par la solitude, léchec et la mort, présentent au moins trois déclencheurs de leur désastre intime, idéologique et social. Ce qui les distingue au départ, ce sont leurs origines et leurs différents tempéraments, mais l'action unique des facteurs idéologiques et socio-politiques approfondissent l'impuissance physique et mentale de Florent (que soulignent les antidépressifs) et brisent la force mentale et socio-économique d'Aymeric. Nous nous proposons donc de montrer que ces deux vies révèlent, chacune à sa manière, la triple castration de l'homme occidental : pharmaceutique (chez Florent), idéologique et socio-politique. En proposant une triple castration de l'homme occidental de notre époque, nous restons pleinement consciente de

19 Sous idéologie nous entendons toute Weltanschauung, sans aucune connotation négative. 
l'impossibilité de séparer les sphères idéologique et socio-politique. Leur traitement séparé dans ce travail n'est qu'une question d'ordre d'analyse.

\subsection{Castration pharmaceutique}

\subsubsection{Sérotonine}

Le roman souvre sur une phrase emblématique, répétée intégralement à la fin du roman : C'est un petit comprimé blanc, ovale, sécable. Ce comprimé - c'est le médicament antidépresseur que Florent prend pour élever son taux de sérotonine, "hormone du bonheur", dans le sang : Plus spécifiquement, la sérotonine était une hormone liée à l'estime de soi, à la reconnaissance obtenue au sein du groupe [...] on signalait son existence chez de très nombreuses créatures vivantes, $y$ compris les amibes. (94)

Par rapport aux PE et à la PI, la place du discours (pseudo-)scientifique dans Sérotonine est minime dans le sens quantitatif (de courtes explications du fonctionnement de la sérotonine et des médicaments inhibant sa recapture par les neurones $5-H T_{1}$, pp. 12 et 94), mais sa position est cruciale - dans le titre même. Comme beaucoup d'hommes de la société occidentale (et de femmes, il s'agit d'une pandémie déjà), le narrateur souffre du manque de sérotonine, ce qui produit chez lui dépression, anxiété, passivité, manque de confiance en soi. Suffira-t-il donc de relever le taux de cette hormone, comme semblent l'avoir prouvé les expériences avec les célèbres homards de Jordan Peterson, professeur de psychologie clinique à Toronto ? Un homard avec un haut niveau de sérotonine et peu d'octopamine devient trop sûr de lui. Il aime à plastronner, et il est peu probable qu'il se retrouve sur le dos lorsquion le défie. ${ }^{20}$

2012 règles pour une vie (2018), chap. 1, sec. " Neurochimie de la défaite et de la victoire ». 
Or, contrairement à l'avis de Peterson, pourtant spécialiste en la matière - pour qui les médicaments qui inhibent la chute du taux de sérotonine " ont un effet chimique et comportemental très semblable " à la sérotonine produite par le corps - ce " petit comprimé blanc, ovale, sécable» agit autrement sur Florent : il provoque son impuissance sexuelle et même le manque de désir. En réalité, il y a là un circle vicieux pour les hommes et femmes dépressifs : la dépression cause déjà de sérieux problèmes sexuels, psychologiques et physiologiques; certains types de médicaments antidépresseurs ne font que les aggraver. ${ }^{21}$ Car ces médicaments ne traitent que les manifestations d'un mal plus profond, qui nest pas dordre physiologique et contre lequel la science est impuissante. Le coeur mal aimé et mal aimant a ses souffrances que les médicaments ne guérissent point. La sérotonine a une place importante en psychologie et sociologie aussi, de sorte que Peterson étudie son influence sur le comportement social des hommards (et des hommes). Dans les livres de Peterson et de MH, nous avons isolé plusieurs sémès autour de la sérotonine :

MH, Sérotonine - /antidépresseur/, /estime de soi/, / reconnaissance par le groupe/.

Peterson, 12 règles pour une vie ${ }^{22}$ - section "Conflit et territoire ": /arrogant/, / se pavane/, /comme Clint Eastwood dans un western spaghetti/, /sétend/, /avance/, /

21 "And it is of course true that pharmacologists are producing a great many new wonder drugs where the cure is almost worse than the disease. Every year the new edition of medical textbooks contains a longer and longer chapter of what are iatrogenic diseases, that is to say diseases caused by doctors (laughter\} And this is quite true, many of the wonder drugs are extremely dangerous. I mean they can produce extraordinary effects, and in critical conditions they should certainly be used, but they should be used with the utmost caution. » A. Huxley, Ultimate Revolution, 1962.

22 Nous avons utilisé le text originel anglais accessible sur https:// archive.org. 22.9.19. 
se bat plus, et plus longtemps/, /victorieux/; section « Haut et bas » :/dominance/, /valeur/...23

La sérotonine est donc le synonyme de la réussite sexuelle et sociale. Et si le niveau de sérotonine est bas ? Peterson de répondre :

Considérez la sérotonine, le produit chimique qui régit la posture et lévasion chez le homard. Les homards en bas de léchelle produisent des niveaux relativement bas de sérotonine. Ceci vaut également pour les êtres humains de bas rang (et ces niveaux bas diminuent davantage à chaque défaite). Linsuffisance de sérotonine implique la perte de confiance. Linsuffisance de sérotonine implique une plus grande réactivité face au stress et une préparation physique plus coûteuse aux situations d'urgence - car tout peut arriver, à tout moment, au bas-fond de la hiérarchie de dominance (et rarement quelque chose de bon). L'insuffisance de sérotonine signifie moins de bonheur, plus de douleur et d'anxiété, plus de maladies et une durée de vie plus courte - autant chez les humains que chez les crustacés. (chap. 1, section « Nature de la nature »; nos italiques et traduction)

C'est la portrait juste de Florent - et de la déchéance d'Aymeric. La prise du petit comprimé blanc suit immédiatement le rituel matinal café-cigarette, à propos duquel Florent (alias $\mathrm{MH}$, grand fumeur) fournit un commentaire profond : La nicotine est une drogue parfaite, une drogue simple et dure, qui napporte aucune joie, qui se définit entièrement par le manque, et par la cessation du manque.(9)

23 Section «Conflict and Territory » : / »cocky »/, / »strutting »/, / »like Clint Eastwood in a spaghetti Western $» /$, / »stretch out $» /$, /advance ", " fight longer and harder ", "victor »; section " Top and Bottom » : "dominance ", "value ». Les deux livres ont été publiés la même année. $\mathrm{MH}$ a pu suivre les conférences de JP sur internet, mais il n'a pu lire Les 12 règles avant la parution de Sérotonine. En tout cas, leurs positions se ressemblent beaucoup. 
Aucune joie, juste un soulagement temporaire, chewinggum pour les affamés. Suit la prise du Captorix, qui produit un plus fort engourdissement du manque, sans aucune joie non plus. Nous retrouvons ici la clé de l'interprétation, en antiphrase, du titre : le traitement pharmaceutique de la souffrance humaine anesthésie la douleur, pour un petit peu ; or, en causant l'impuissance sexuelle, le Captorix empêche la seule joie qui compte pour les hommes de $\mathrm{MH}$, celle quapporte le sexe. Et pourtant, ce profond mal dêtre, contre lequel les médicaments sont si impuissants, disparait dès qu'un simple amour sincère remplit lêtre d'une joie débordante. Le baiser au lépreux qui opère le miracle. Alors que l'idéal moderne du «bonheur » voit dans le monde l'instrument de son propre plaisir insatiable. Est-ce pour cela que [p]lus personne ne sera heureux en Occident [...] plus jamais, nous devons aujourd'hui considérer le bonheur comme une rêverie ancienne, les conditions historiques neen sont tout simplement plus réunies? (102) Ces conditions historiques que $\mathrm{MH}$ voit disparaître, c'est l'incarnation, dans les hommes et femmes prêts à aimer au prix du risque de la souffrance, de cette idéologie qui retrouve le bonheur dans le don de soi.

\subsubsection{Soma}

La titre même de Sérotonine souligne la castration pharmaceutique de Florent, la plus explicite dans le roman consistant à rendre l'homme sexuellement impuissant par le médicament même qui devrait lui rendre la virilité et le contrôle de sa propre vie. Par sa promesse du bonheur, et même par sa forme, ce petit comprimé rappelle le soma ${ }^{24}$

24 L'espace nous manque pour étudier le parallèle entre le contre-effet du Captorix sur Florent et la lobotomie finale dans le roman Nous autres $(\mathrm{Mbl}, 1920)$ de Ievgeni Zamiatine. 
du Le meilleur des mondes (1932), ${ }^{25}$ drogue « [e] uphorique, narcotique, agréablement hallucinant [e] » (chap. 3), qui garantit la stabilité de l'État mondial :

Et si jamais, par quelque malchance, il se produisait d'une façon ou d'une autre quelque chose de désagréable, eh bien, il y a toujours le soma qui vous permet de prendre un congé, de vous évader de la réalité. Et il y a toujours le soma pour calmer votre colère, pour vous réconcilier avec vos ennemis, pour vous rendre patient et vous aider à supporter les ennuis. Autrefois, on ne pouvait accomplir ces choses-là qu'en faisant un gros effort et après des années d’entraînement moral pénible. À présent, on avale deux ou trois comprimés d'un demi-gramme, et voilà. (chap. 17) ${ }^{26}$

Le rôle du soma, que tous les citoyens de l'État mondial sont censés prendre pour "se reposer d'eux-mêmes ", ${ }^{27}$ est donc avant tout d'assurer la stabilité de l'ordre social, non plus avec la violence et la terreur, mais en anesthésiant le manque ontologique humain (qui produit dans l'homme la frustration et l'ennui capables d'engendrer en révolte collective) par la saturation superficielle, immédiate et incessante des désirs physiques. La conscience morale et les passions profondes sont ainsi étouffées, les questions existentielles écartées. Toute la société, plongée dans les plaisirs sensoriels propres aux « kids définitifs » (La possibilité d'une île, 38), est devenue un camp

25 Le meilleur des mondes figure déjà comme intertexte dans Les particules élémentaires (1998), notamment dans le chapitre intitulé " Julian et Aldous».

26 http://docplayer.fr/25228943-Aldous-huxley-le-meilleur-desmondes-brave-new-world-1932-traduction-de-jules-castier.html 10.10.2019.

27 Comme le dit Huxley dans La révolution ultime, " man has always had a hankering after mind changing chemicals, he has always desired to take holidays from himself $»$. 
de concentration indolore (painless concentration camp). ${ }^{28}$ Le Captorix de Sérotonine fonctionne de manière semblable, et bien que $\mathrm{MH}$ ne mentionne pas explicitement son impact social, il se voit dans les commentaires de Florent : l'utilité de ce médicament était indéniable, grâce à lui ma vie sociale était maintenant dénuée de heurts... (154). L'homme anesthésié par l'antidépresseur ne peut pas participer activement dans l'action sociale, c'est « un grand corps malade » impuissant. Le nom même de soma réunit deux concepts religieux bien différents. Dans les Védas, soma, mot sanscrit, désigne une sorte d'ambroisie que les humains offraient aux dieux, et dont la consommation garantissait l'immortalité. Ce parallèle suggère le statut pseudo-divin des habitants de l'État mondial ( $\mathrm{O}$ brave new world, that has such people in it ") ${ }^{29}$ qui vivent sans soucis, immergés dans des plaisirs sensoriels incessants. De l'autre côté, le soma rappelle étrangement la Cène, dernier repas que le Christ a partagé avec ses disciples à la veille de sa crucifixion. Dans la célèbre formule : «Prenez, mangez, ceci est mon corps " (Matthieu, XXVI, 26 ; trad. Ostervald), corps traduit le mot grec soma $(\sigma \tilde{\omega} \mu \alpha)$. Si le soma védique représente l'offrande des hommes aux dieux, le soma chrétien désigne l'offrande de Dieu aux hommes. De même, le soma chez Huxley est la véritable eucharistie de l'État mondial, leur «pain de vie » - garant de leur béatitude, la colle sociale, le facteur de la stabilité de l'individu et de la société. De la sorte, si léternité des chrétiens est fondée sur le sacrifice suprême

28 A. Huxley, discours de Tavistock Group, California Medical School, 1961.

29 Ce célèbre vers de la Tempête de Shakespeare est cité en exergue du texte anglais du Meilleur des mondes, que les traducteurs français ont remplacé par la phrase équivalente du Candide « Tout est pour le mieux dans le meilleur des mondes. » 
de Jésus-Christ, la fausse éternité de l'État mondial est fondée sur l'absence de souffrance.30 Dans Sérotonine, le petit comprime de Captorix anesthésie le consommateur, sans aucune grâce divine. Tandis que la Bible peint l'homme créé « à notre image, selon notre ressemblance » (Gen. I, 26, trad. Osterval), économe de Dieu (oíkovónoc ; par ex. Luc XII,42) sur la Terre entière, les produits chimiques qui soulagent la souffrance psychique de l'homme dans ce monde déshumanisé ont un effet contraire - ils rendent l'homme déchu incapable de contrôler sa propre vie.

Rendant tous les humains contents de leur servitude, le soma est le garant de la stabilité sociale. Or, comme le montre le mécontentement de Bernard Marx, ainsi que le conflit entre l'État mondial et Jean le Sauvage (modelé sur le Jean-Baptiste biblique), le plaisir n'est pas toujours le bonheur, surtout lorsqu'il ne résulte pas du love's labour (peines d’amour). Dans la célèbre conférence sur La révolution ultime (Ultimate Revolution, Berkeley, 1962), Huxley avertit contre la manipulation par le plaisir suscité par les drogues:

Dans les mains d'un dictateur, ces substances, d'une manière ou autre, pourraient d'abord être utilisées sans aucun dommage, et le résultat serait, comme vous pouvez l'imaginer, l'euphorie qui rendrait les humains complètement heureux dans les circonstances les plus abominables. [...] C'est-à-dire, il y plusieurs années de cela, un collocataire m’a dit, après avoir lu Le paradis perdu de Milton : 'Et la bière fait plus que Milton pour justifier les voies de Dieu aux hommes.' Alors que la bière est une drogue extrêmement grossière comparée à celles-ci. Et vous pouvez dire

30 Timothy Leary nommera les Beatles, en 1967, « les quatres apôtres » d'un tout nouveau testament : " [T] he message from Liverpool is the Newest Testament, chanted by Four Evangelists - saints John, Paul, George, and Ringo.» V. The Gospel According to the Beatles de Steve Turner, John Knox Press, Louisville, KE, 2006. 
avec sûreté que certains stimulants psychiques et certains hallucinogènes pourraient accomplir bien plus que Milton et tous les théologiens ensemble pour rendre plus supportable qu'il ne l'est le mystère terrifiant de notre existence. [...] Agréable au point où, comme je l'ai déjà dit, les Humains finiraient par aimer l'état des choses que, selon tous les standards raisonnables et décents, ils ne devraient pas aimer, et je crois que c'est entièrement possible. ${ }^{31}$

Le roman commence et se termine par le petit comprimé blanc; ses consommateurs sont pris dans le cercle infernal de l'impuissance d'aimer et de créer, deux propriétés les plus divines de l'homme. Dans ce soma de notre époque se résume le paradoxe de la condition humaine : à fuir la souffrance, on fuit aussi la capacité du bonheur. Du même coeur nous aimons et nous souffrons. Soma ou Captorix, la joie synthétique rend impuissant et dévasté. " And love, so distant et obscure, remains the cure. ${ }^{32}$

31 "In the hands of a dictator these substances in one kind or the other could be used with, first of all, complete harmlessness, and the result would be, you can imagine a euphoric that would make people thoroughly happy even in the most abominable circumstances. I mean these things are possible. [...] I mean, a housemate years ago remarked after reading Milton's Paradise Lost, He Says "And beer does more than Milton can to justify God's ways to man" (laughter). And beer is of course, an extremely crude drug compared to these ones. And you can certainly say that some of the psychic energizers and the new hallucinants could do incomparably more than Milton and all the Theologians combined could possibly do to make the terrifying mystery of our existence seem more tolerable than it does. [...] Enjoyable to the point, where as I said before, Human beings come to love a state of things by which any reasonable and decent human standard they ought not to love and this I think is perfectly possible. » (notre traduction) https://publicintelligence.net/aldoushuxley-1962-u-c-berkeley-speech-on-the-ultimate-revolution/ 16.9.2019.

32 Eric Carmen, « All by Myself », 1975. 


\subsection{Castration idéologique}

Sérotonine confronte deux idéologies opposées : d'un côté le libéralisme (moral et économique), qui imprègne toute la société ; de l'autre le conservatisme (pro)chrétien, se manifestant plus discrètement, mais avec plus de fermeté que dans Soumission.

\subsubsection{Libération sexuelle et féminisme}

L'influence désastreuse de la libération sexuelle dans la deuxième moitié $\mathrm{du} \mathrm{XX}^{\mathrm{e}}$ siècle sur la définition du bonheur pour plusieurs générations fait un des grands topoï houellebecquiens. Fils d'une libertaire égoïste à qui il n'a jamais pardonné de l'avoir confié à sa grand-mère paternelle dont l'amour, si grand soit-il, n’a jamais pu cicatriser la plaie béante du désamour maternel, $\mathrm{MH}$ développe dans plusieurs romans un vaste panorama de la transformation profonde de l'Occident. Dans Sérotonine, il résume les conséquences des illusions de liberté individuelle, de vie ouverte, de l'infini des possibles (347). Son analyse laisse penser que le pacifisme de "Make love not war ", sopposant aux guerres entre nations, coïncide ironiquement avec un belliqueux « Make war not love » de ce féminisme dont le combat juste pour l'égalité des sexes a dégénéré en une guerre acharnée contre l'homme ${ }^{33}$ - dans les institutions, et au sein des familles, rendant désamour pour désamour :

Il est piquant de constater que cette libération sexuelle a parfois été présentée sous la forme d'un rêve communautaire, alors que le beau mot de 'ménage', le couple et la famille représentaient le dernier îlot de communisme primitif au sein

33 Il suffit de penser aux concepts aberrants derrière les néologismes tels mansplaining ou même manspreading. 
de la société libérale. La libération sexuelle eut pour effet la destruction de ces communautés intermédiaires, les dernières à séparer l'individu du marché. Ce processus de destruction se poursuit à nos jours. ${ }^{34}(\mathrm{PE}, 116)$

Pour Christiane des PE, quoiqu'épouse délaissée pour une plus jeune, les féministes

n'arrêtaient pas de parler de vaisselle et de partage des tâches ; elles étaient littéralement obsédées par la vaisselle. Parfois elles prononçaient quelques mots sur la cuisine ou les aspirateurs ; mais leur grand sujet de conversation, cétait la vaisselle. En quelques années, elles réussissaient à transformer les mecs de leur entourage en névrosés impuissants et grincheux. (PE, 146, nos italiques)

Pas seulement les mecs; le désamour (des hommes) rendra les femmes tout aussi névrosées et grincheuses. Progressivement, le ménage devient la lice où lui et elle combattent pour arracher de l'autre sa part de jouissance. Au lieu de l'amour fusionnel, les particules élémentaires. Serait-ce le sens final du célèbre "Freedom's just another name for nothing left to loose » ? ${ }^{35}$ Comme le montre l'analyse de $\mathrm{MH}$ - ainsi que les désastres intimes tout autour de nous - la carence émotive produit un profond manque de paix, de stabilité, de joie - d'où la frustration et la dépression. Ne faudrait-il donc pas reconnaître dans l'obsession sexuelle de l'homme houellebecquien une déviation issue du manque d'amour fondamental et donc indéracinable, " rectifiée " par la castration émotive, idéologique, puis socio-politique et, coup de grâce, pharmacologique de cette virilité diaboli-

34 Comme pour conclure ce processus de destruction depuis 1998, le narrateur insiste : Il aurait fallu graver cette phrase, en lettres énormes, sur tous les édifices publics : l'abandon de la famille, en France, ne constitue pas un délit. (59)

35 Paroles de la chanson "Me and Bobby McGee » (Chris Christopherson), chantée notamment par Janis Joplin. 
sée ? Toujours est-il que l'accusation explicite de misandrie ne figure guère dans Sérotonine (à part cette remarque sur les hommes qui « avaient été détruits par les femmes occidentales, le cas le plus flagrant étant en effet celui des Anglo-Saxonnes », 156, 157). Au contraire, l'amour de Kate et Camille pour Florent, cette puissance génératrice qui crée un monde nouveau (70), représente des signes extrêmement clairs (347) du don divin d'amour qu'il faut accepter et soigner, amour que l'indifférence criminelle de Florent laisse aller.

La corrélation idéologie-société-individu est explicite lorsque Florent essaie d'expliquer pourquoi il a laissé partir Camille, l'amour de sa vie, au moment où elle quittait Rouen pour poursuivre ses études à Paris :

J'étais heureux, jamais je n'avais été aussi heureux, et jamais plus je ne devais l'être autant [...] J'aurais pu lui proposer d'arrêter ses études, de devenir femme au foyer, enfin de devenir ma femme, et avec le recul quand j'y repense [...], je pense qu'elle aurait dit oui ! [...] Mais je ne l'ai pas fait et sans doute je ne pouvais pas le faire, je navais pas été formaté pour une telle propositions, ça na faisait pas partie de mon logiciel, jétais un moderne, et pour moi comme pour tous mes contemporains la carrière professionnelle des femmes était une chose qui devait être avant toute autre respectée, cétait le critérium absolu, le dépassement de la barbarie, la sortie du Moyen Âge [...] il me semble que j’avais déjà peur, et que j’avais bien compris, déjà à cette époque, que le monde social était une machine à détruire l'amour. (172,173, nos italiques)

Le destin du couple relie ainsi les plans social, idéologique et individuel : il y a amour et désir, mais Florent n’ose pas suivre son désir d'épouser Camille de peur d'empêcher sa carrière, parce qu'il y a culpabilisation du désir de l'homme en tant qu'entrave pour l'émancipation de la femme. C'est une sorte de conditionnement par de nouvelles normes, toujours menaçant de montrer l'homme de 
doigt. La carrière devient un objectif idéologiquement et socialement valorisé plus que le couple. Désormais, réussite rime avec solitude. De là cette question finale de Florent :

Avons-nous cédé à des illusions de liberté individuelle, de vie ouverte, d'infini des possibilités? Cela se peut, ces idées étaient dans l'esprit $d u$ temps; nous ne les avons pas formalisées, nous n'en avions pas le goût; nous nous sommes contentés de nous y conformer, de nous laisser détruire par elles; et puis, très longuement, d'en souffrir. (347)

L'impératif de liberté individuelle a fini par isoler ceux qui auraient pu saimer dans leurs petits royaumes sans bonheur. Bruno Viard explique l'équation houellebecquienne entre " libéralisme économique " et " libéralisme sexuel », déjà développée dans l'Extension du domaine de la lutte:

Dans les deux cas, la loi de l'offre et de la demande produit les mêmes effets, c'est-à-dire paupérisation des perdants et clivage de la société en winners et losers. Certains sont gagnant/ gagnant, d'autres sont perdant/perdant, d'autres enfin sont gagnant/perdant ou perdant/gagnant. Les héros de Houellebecq, eux, ont tous un niveau économique et intellectuel satisfaisant, mais au plan sexuel ce sont des perdants. [...] Le sujet du romancier, c'est le sort des exclus de la société moderne en général, mais c'est dans l'exclusion sexuelle qu'il s'est spécialisé. La fin du mariage et de la famille signifie une vie sexuelle intense et variée pour les gagnants, mais la solitude, la frustration et le repli sur la masturbation pour les perdants. On voit bien où serait l'idéal dans ces conditions : le collectivisme en économie et une vie conjugale et familiale stable en matière sexuelle.» (Viard, $2010: 171$; ses italiques)

Le désastre intime et social de Florent (et d'Aymeric) n'est donc pas seulement dérivé d'une personnalité veule ou incapable mais, en dernière instance, d'une terrible utopie 
idéologique comprenant la fausse promesse d'un bonheur impossible de deux êtres égocentriques qui se disputent tout brin de plaisir. ${ }^{36} \mathrm{Car}[i] l$ ny a pas d'amour dans la liberté individuelle, dans l'indépendance [...] il n'y a d'amour que dans le désir d'anéantissement, de fusion, de disparition individuelle, dans une sorte [...] de sentiment océanique (PI, 389 , italiques supprimés).

\subsubsection{Conservatisme et christianisme}

La position de MH dans Sérotonine est nettement identitaire et souverainiste. Elle est conservatrice dans les sens politique et religieux.

D'un côté, il loue dans la personne d'Aymeric les valeurs et l'histoire d'une France aristocratique, la France dont la force étaient ses héros viriles. En étudiant les ancêtres d'Aymeric, Florent se dit impressionné par le nombre d'ambassadeurs, de prélats et de chefs militaires qu'ils avaient donnés au pays (271). Incarnant une telle France, vouée à la disparition, Aymeric d'Harcourt-Olonde, l'aristocrate et grand propriétaire terrien, jouit du respect des villageois, surtout après sa mort. Qui plus est, dans un café non loin du château d'Aymeric règne une atmosphère presque Ancien Régime, comme si 1789 n'y avait laissé que des traces superficielles (269). Un autre indice éloquent : après la tragédie d'Aymeric et de ses partisans, seul le Rassemblement national semblait tout à fait clair sur ce sujet $(265) .^{37}$

36 Ainsi nous avons vu que Tristan aime Iseut non point dans sa réalité, mais en tant qu’elle éveille en lui la brûlure délicieuse du désir. Lamour-passion tend à se confondre avec l'exaltation d'un narcissisme... » (D. de Rougemont, L'Amour et l'Occident, III, 3)

37 Rappelons, à ce sujet, sa position politique dans Soumission : «Le 
De l'autre côté, nous reconnaissons dans Sérotonine un nombre important d'allusions au christianisme, notamment dans sa version catholique, comme antidote contre l'anomie de la société et la désorientation de l'individu.Or, lesprit $d u$ temps ne rime nullement avec les valeurs chrétiennes: le troisième millénaire venait de commencer, et ceétait peut-être, pour l'Occident antérieurement qualifié de judéo-chrétien, le millénaire de trop (102-103). Même si MH se dit incapable de croire, ses sympathies pour certaines valeurs catholiques et chrétiennes - telles fidélité, sacrifice, famille, patrie sont évidentes, même si parfois subtiles: Il était deux heures du matin, la nuit nén était qu'à son mi-temps, cétait l'heure de loffice de vigiles, dans les monastères (279) ; les moines, par exemple, sécrètent très peu de cortisol (320, en parlant du stress). Il évoque [l]es flèches de la cathédrale [...] de Coutances, lesquelles sont d'une grande élégance (270). Aucune ironie ici, mais le respect d'une foi authentique. Déjà dans la PI, Daniel explique pourquoi il ne peut pas se moquer de la foi sincère : lorsqu'ils [les êtres humains] donnent [...] l'impression dêtre animés d'une foi profonde, par quelque chose qui outrepasse l'instinct de survie, le mécanisme grippe, le rire est arrêté dans son principe (221).

Plusieurs fois, malgré l'absence d'irrévérence, un certain vide sémantique et émotif accompagne l'usage des expressions religieuses partiellement figées : demain s'occupera de demain, comme dit à peu près l'Ecclésiaste (266); tel était le destin des femmes, elle le savait, jusqu’à l'avènement $d u$ Christ en gloire (24); car Dieu a permis la parure (24); elle sécraserait directement cent mètres plus bas, un moment de terreur pure et puis ce serait fini, je rendrais au Seigneur mon âme incertaine (28). Face à ces expressions vides de 
conviction, nous soulignons deux références à Dieu comme vrai. Dans la première, Dieu existe, mais il est responsable du malheur humain :

Dieu est un scénariste médiocre, c'est la conviction que presque cinquante années d'existence m’ont amené à former, et plus généralement Dieu est un médiocre, tout dans sa création porte la marque de l'approximation et du ratage, quand ce nest pas celle de la méchanceté pure et simple [...] il y a forcément des exceptions, la possibilité du bonheur devait subsister ne fût-ce quà titre d’appât (181)

La deuxième fois, à la fin du roman, après avoir longtemps repensé sa vie, Florent a compris qu'il a ignoré les directives et les signes de Dieu, et que sa désolation n'est que de sa propre faute :

Dieu soccupe de nous en réalité, il pense à nous à chaque instant, et il nous donne des directives parfois très précises. Ces élans d’amour qui affluent dans nos poitrines jusquà nous couper le souffle, ces illuminations, ces extases, inexplicables si lon considère notre nature biologique, notre statut de simple primates, sont des signes extrêmement clairs.

Et je comprends, aujourd'hui, le point de vue du Christ, son agacement répété devant lendurcissement des coeurs : ils ont tous les signes, et ils nen tiennent pas compte. Est-ce qu'il faut vraiment, en supplément, que je donne ma vie pour ces minables ? Est-ce qu'il faut vraiment être, à ce point, explicite?

\section{Il semblerait que oui. (347)}

$\mathrm{MH}$ pose ici, comme en conclusion de tous ses romans, cette question fondamentale, qui n'est pas seulement celle du Christ dans le jardin de Ghetsémani, mais celle de chaque être humain confronté avec la douleur que lui coûte d'aimer ses semblables : faut-il que je donne ma vie pour ces minables? Autrement dit, l'amour vaut-il ses peines? 
La réponse est au conditionnel, mais elle est définitive : Il semblerait que oui. Car [l]e monde extérieur était dur, impitoyable aux faibles, il ne tenait presque jamais ses promesses, et l'amour restait la seule chose en laquelle on puisse encore, peut-être, avoir foi. (180) Sérotonine est donc, plus que tous les romans précédents, la confession de la seule foi dont soit capable $\mathrm{MH}$, la foi en l'amour.

\subsection{Castration socio-politique}

Après les contextes de beaux-arts, sciences, tourisme, politique et religion dans les romans précédents, Sérotonine discute l'effondrement de la agriculture française en temps réel. Ingénieur d’agronomie (comme $\mathrm{MH}$ ) ayant travaillé au ministère de l'Agriculture, Florent développe un discours bien moins cultivé et plus spécialiste. Il commente la politique agro-alimentaire de l'Union européenne - dont il nous rapporte les décisions qui, avec les quotas, la baisse des prix et la politique du libre échange, vouent à l'incompétitivité, et donc à la banqueroute finale, des miliers d'agriculteurs et éleveurs français : ${ }^{38}$

Le nombre d’agriculteurs a énormément baissé depuis cinquante ans en France, mais il na pas encore suffisamment baissé. Il faut encore le diviser par deux ou trois pour arriver aux standards européens, aux standards du Danemark ou de la Hollande [...] Bref, ce qui se passe en ce moment avec l'agriculture en France, c'est un énorme plan social, le plus gros plan social à loeuvre à l'heure actuelle, mais c'est un plan

38 Bien sûr, il en est ainsi dans tous les pays de l'Union européenne: Comme tous les pays de l'Europe occidentale, l'Espagne, engagée dans un processus mortel d'augmentation de la productivité, avait peu à peu supprimé tous les emplois non qualifiés qui contribuaient jadis à rendre la vie un peu moins désagréable, condamnant du même coup la majeure partie de sa population au chômage de masse. (26) 
social secret, invisible, où les gens disparaissent individuellement, dans leur coin [...] une fois qu'on sera aux standards européens, on n'aura toujours pas gagné, on sera même au seuil de la défaite définitive, parce que là on sera vraiment au contact avec le marché mondial, et la bataille de la production mondiale on ne la gagnera pas. (248-249)

Ici, la fiction cède la place à la réalité. $\mathrm{MH}$ peint avec amertume non seulement la détresse de plus en plus criante des agriculteurs de sa région (186), mais celle de la civilisation française entière. Par exemple, Florent parle à une femme qui va vendre la maison paternelle aux Hollandais (175), et dont, en plus, lépoux venait de perdre son emploi chez [l'entreprise] Graindorge, [après] son rachat par Lactalis, le numéro un mondial du lait. En écoutant cette histoire, il se dit : javais échoué à aider l'entreprise de son mari et finalement à sauver son emploi (176). Sur ce point, Florent se montre incapable de protéger les intérêts des agriculteurs français : ${ }^{39}$

« définir, soutenir et représenter les positions de l'agriculture française » [...] je ne pouvais qu'aligner une impressionnante succession d'échecs dans ma défense des positions agricoles de la France, mais ces échecs nétaient pas les miens, ils étaient bien plus directementt ceux des conseillers négociateurs, espèce rare et vaine dont les insuccès répétés n'entamaient nullement la morgue (30)

Par son attitude impuissante et indifférente, il trahit même son ami Aymeric, producteur laitier : jétais bien obligé de me rendre compte que jétais maintenant du côté de

39 Par la bouche du narrateur, $\mathrm{MH}$ affirme qu'il fallait au contraire privilégier la qualité, consommer local et produire local, protéger les sols et les nappes phréatiques en revenant à des assolements complexes et à l'utilisation des fertilisants animaux. (109) 
l'Etat français, que nous n’étions plus tout à fait dans le même camp (152).

La convergence des domaines idéologique et socio-politique devient particulièrement intense et complexe dans le destin d'Aymeric, le meilleur ami de Florent. Car, si Florent est dès le début un perdant, Aymeric a, au début, tous les traits d'un chevalier médiéval "sans peur et sans reproche ", qui ne souffre nullement d'un quelconque manque de sérotonine. Si Florent est traitre (selon $\mathrm{MH}$ ) de la femme, de la France et de sa profession, Aymeric est un héros. Artistocrate de souche, sérieux et travailleur, il fait, après ses études d'agronomie, un stage chez Danone, la plus grande multinationale française agroalimentaire, mais refuse d'y faire une carrière en choisissant, le seul de sa promotion, de devenir agriculteur. Sur les terres de ses ancêtres, il élève 300 vaches laitières : J'essaie de faire les choses correctement, ça n'a rien d'élevage industriel ici [...] Mais plus jessaie de faire les choses correctement, moins jarrive à men sortir. (150) Aymeric s'adresse à l'Etat afin d'obtenir de l'aide pour restaurer son "vieux château presque délabré » (144, citation de Barbey d'Aurevilly), sans succès. L'économie française ne favorise pas la production locale ; la vie devient dure et Aymeric est obligé de vendre une partie de ses terres aux étrangers $(145,146$. Comme lui, de nombreux autres agriculteurs (correspondant tous au même type d'homme qu'Aymeric, forts, virils et courageux) n'arrivent pas à rembourser leurs emprunts (Rien que d'employer le mot «comptabilité » avait suffi à l'assombrir, 150), alors que l'Etat ne protège ni leurs intérêts ni leurs produits, ce qui les pousse à une rébellion ouverte, surtout après plusieurs suicides de leurs collègues. Malgré un énorme travail quotidien, Aymeric souffre de manquer à ses devoirs : Moi jessaie de monter quelque chose, je me crève au boulot, je me lève tous les jours à cinq heures, je passe mes soirées dans la comptabilité - et 
le résultat, en fin de compte, ceest que jappauvris la famille... (226) La situation socio-politique affecte la vie intime d'Aymeric : sa femme craque sous ce poids (je l'ai trop fait bosser [...] jaurais dî la ménager [...] Les femmes, ça a besoin des vacances..., 204), en emmenant les filles avec elle, et l'homme ruiné reste seul : il était juste en train de crever de leur absence, et de l'absence d'amour plus généralement [...] il était foutu [...] il ne sortirait jamais de cette histoire, il en souffrirait jusqu’à la fin de ses jours (222).

Ainsi, ce gagnant devient perdant, et $\mathrm{MH}$ présente sa descente aux enfers comme directement et uniquement causée par les mesures économiques désastreuses de l'État français et de l'Union européenne. Peu à peu, une spirale d'anéantissement et de mort (254) rapproche Aymeric, morose, buté et désespéré (229), de l'état mental de Florent, vieux mâle vaincu (210) : Ses traits étaient épaissis et couperosés, mais c'est surtout le regard qui était effrayant, un regard creux, mort, qu'il semblait impossible de distraire, davantage que quelques secondes, de la contemplation du vide. (195) Le divorce d'Aymeric implique également un contexte légal - la ruine supplémentaire et la séparation entre père et enfants :

Si Cécile sobstinait à réclamer la moitié de la valeur des bungalows, ils nauraient d'autre choix que de mettre lentreprise en liquidation judiciaire [...] votre état d'esprit permanent lors d'un divorce à mesure que se met en place la procédure, que se succèdent les tractations, négotiations, propositions et contre-propositions d'avocats et notaires, en somme ce divorce, il nétait pas près d’en voir la fin. (225)

Les filles d'Aymeric seront élevées par l'amant de leur mère, un pianiste anglais : on était dans le dur, dans l'humiliation masculine à létat brut (206); est-ce qu'un pianiste de concerts classiques ça pouvait leur donner une image 
paternelle virile [...] alors que leur père avait affaire à des vaches adultes, de gros mammifères tout de même, au moins cinq cents kilos (222). La souffrance d'Aymeric, trahi par la femme et par la Patrie, se soldera par le suicide de cet " aristocrate martyr de la cause paysanne " (268). Le portrait d'Aymeric au paroxisme de sa révolte contraste avec la misère morale de Florent :

Déjà il était souverainement beau, les bouffisures de son visage semblaient mystérieusement annulées, et surtout il paraissait paisible, amusé presque, sa longue chevelure blonde flottant dans un souffle de vent qui sétait, à cette seconde, levé [...] il tenait à demi-dressé, contre sa hanche, son fusil d'assaut Schmeisser [...] à cette seconde Aymeric paraissait heureux, enfin presque heureux, il paraissait à sa place tout du moins, son regard et sa pose décontractée surtout reflétaient une incroyable insolence, il était l'une des images éternelles de la révolte (258)

Cet Aymeric beau, droit, libre et insoumis, qui a remplacé l'épée par un Schmeisser pour combattre l'injustice et défendre son peuple, c'est cet éternel combattant contre le mal et l'injustice - et c'est pourquoi, au moment où il revendique sa liberté et son bonheur perdu, Aymeric paraissait heureux [...] à sa place tout du moins. Mais un homme pleinement conscient de son identité ne peut pas survivre dans l'Etat qui se tourne contre ses hommes les plus vaillants; le suicide d'Aymeric est un terrible cri de protestation contre les eurocrates (182) et d'inutiles marionnettes européennes (333) qui écrasent « la France de Johnny » :

il avait simplement voulu être heureux, il sétait engagé dans son rêve agreste basé sur une production raisonnable et de qualité [...] et l'Union européenne elle aussi avait été une grosse salope, avec cette histoire des quotas laitiers, il ne s'attendait 
certainement pas à ce que les choses se terminent ainsi. (259, nos italiques)

Ayant ainsi perdu son dernier proche, Florent, incapable d'apprendre de ses erreurs, se retire complètement de la société : je ne parvenais simplement plus à assumer la complexité du monde où jétais plongé (290).

\section{Conclusion}

En plus du portrait houellebecquien classique du solitaire involontaire, Sérotonine développe l'analyse d'une véritable castration psychologique, pharmacologique, idéologique et socio-politique de l'homme européen hétérosexuel d'âge moyen.

$\mathrm{Au}$ niveau pharmaceutique, le profond mal d'être de l'homme occidental est désensibilisé par les médicaments qui tuent à la fois la douleur et la joie de vivre. Sous thérapie, Florent peut endurer son existence vide, mais ne peut pas connaître la réjouissance quapportent la passion amoureuse et la création. Au niveau idéologique, la profonde transformation des règles de communication entre homme et femme rend l'homme incertain dans son approche à la femme et le culpabilise à chaque pas dêtre viril, sexuel, fort, protecteur et dominant. L'homme abandonne alors la partie et perd le nord. Finalement, la situation socio-politique définit les règles - sous la férule de l'idéologie dominante des jeux que l'homme peut jouer en famille, dans la société et dans la production des biens, et cela signifie, pour de plus en plus d'hommes, la déchéance financière, la perte de dignité et d'autorité dans la famille, l'humiliation sociale et l'abandon du sol natal, qui est devenu non rentable. Dorénavant, l'élevage et le paturage ne sont plus les deux 
mamelles de la France. Elle importe le lait étranger, qui est moins cher.

Enfin, bien que traitée ici en première, la castration pharmacologique ne vient quà la fin, après la désorientation idéologique et la faillite politique et économique de l'homme, comme un coup de grâce qui fait oublier sa déshumanisation. Mais si l'homme ne proteste pas, du fond de son abîme, comme l'a fait Aymeric en réclamant sa liberté, ce n'est certainement pas parce que la société et son idéologie libérale l'ont libéré. Libérer l'homme, chaque homme, c'est libérer son eros et sa poiesis, c'est lui permettre d'abord d'être lui-même, c'est lui rendre son autorité, sa dignité et sa mission sur la terre. Pour ce faire, il lui faudra une révolution d'abord spirituelle et personnelle, où il apprendra à vivre avec la douleur d'exister, d'aimer et de créer comme conditio sine qua non de tout bonheur sur Terre.

Sources, bibliographie, discographie

Berger, Sandra. «Les discours (pseudo-)scientifiques dans l’ouvre houellebecquienne ». Muriel-Lucie Clément et Sabine van Wesemael (éds). Michel Houellebecq à la Une. Faut-Titre, $\mathrm{n}^{\circ} 360$, Amsterdam, New York : Brill/Rodopi, 2011. Web 20.10.2019.

Carmen, Eric. «All by Myself». Eric Carmen. Rhino/Arista, 1975. Web 10.10.2019.

Kristofferson, Kris. "Me and Bobby McGee ». Kristofferson.

Washington D. C. : Monument Records, 1970. Web 15.10.2019.

Houellebecq, Michel. Les particules élémentaires. Paris : Flammarion, 1998. Imprimé.

La possibilité d’une île. Paris: Fayard, 2005. Imprimé.

La carte et le territoire. Paris : Flammarion, 2010. Imprimé.

Soumission. Paris : Flammarion, 2015. Imprimé. 
Sérotonine. Paris : Flammarion, 2019. Imprimé.

Huxley, Aldous. Le meilleur des mondes (Brave New World, 1932), trad. Jules Castier, Paris : Plon, 1933. Web 01.09.2019.

Huxley, Aldous. The Ultimate Revolution. Discours prononcé à

l'Université de Berkeley en 1962. Web 16.9.2019.

Jacquin, Joseph (paroles) ; Soulacroix, Jacques (musique). "La chanson du gueux d'amour ». Paris : Ch. Hayet, 1920. Web 12.09.2019.

Peterson, Jordan B. 12 rules for life. An Antidote to Chaos, New York : Penguin Random House, 2018. Web 15.09.2019.

Rastier, François. "Systématique des isotopies ». Essais de sémiotique poétique. Greimas, Algirdas-Julien (éd). Paris : Larousse, 1972. 80-126. Web 04.09.2019.

Rastier, François. "Topoï et interprétation ", Etudes françaises.

Le sens $(d u)$ commun : histoire, théorie et lecture, vol. $36, \mathrm{n}^{\circ} 1$, 2000. 93-107. Web 04.09.2019.

Rougemont, Denis de. L'Amour et l'Occident. Paris : Plon, 1956. Web 01.07.2019.

Самарџија, Татјана. « Постоји једно острво - љубав у поезији Боре Ђорђевића и романима Мишела Велбека ». Језик, книжевност, музика: поезија и музика Боре Ђорђевића (радови са округлог стола одржаног у Андрићграду 7. јула 2018). Милош Ковачевић (ур). Андрићград: Андрићев институт, 2018. 165-193. Imprimé.

Turner, Steve. The Gospel According to the Beatles, Louisville (KE): John Knox Press, 2006. Web 11.08.2019.

Viard, Bruno. "Les enjeux idéologiques de l'oeuvre romanesque de Michel Houellebecq ". Le monde de Houellebecq. Actes du colloque "Le Monde de Houellebecq " tenu les 28 et 29 octobre 2005 à Edimbourg. Gavin Bowd (éd). Glasgow: University of Glasgow, French and German Publications, 2010². 171-184. Imprimé.

Zamiatine, Eugène. Nous autres. Paris : Gallimard, 1971. Web 16.09.2019. 
Татјана Самарџија

\section{ТРОСТРУКА КАСТРАЦИЈА МУШКАРЦА У РОМАНУ СЕРОТОНИН МИШЕЛА ВЕЛБЕКА}

\section{Сажетак}

У раду се разматра трострука кастрација ероса и поесиса (сексуалне и стваралачке снаге) западног мушкарца представљена у роману Серотонин. Бројни цитати, као и учестале лексеме показују да ова кастрација подразумева следеће нивое: фармацеутски (антидепресиви не лече депресију, већ производе импотенцију и појачавају друштвену изолацију појединца), идеолошки (либерална идеологија трансформише мушко-женске односе, што снажно утиче на интимне и породичне односе) и социо-политички (актуелне политичке и правне мере подривају ауторитет мушкарца у породици, обесхрабрују предузетнички дух и слабе везу између човека и родног тла).

Кључне речи: Велбек, Запад, импотенција, кастрација, либерализам, серотонин, феминизам, Хаксли, хетеросексуализам, хришћанство. 